ISSN: 2638-6062

\title{
Experiments about Time and Space
}

\author{
Patrice F Dassonville* \\ Department of physics and chemistry, France
}

Received: 眥 November 30, 2018; Published: 㘹 December 04, 2018

*Corresponding author: Patrice F Dassonville, Department of physics and chemistry, France

\begin{abstract}
It is crucial to find out if time and space are physically existing or not. In other words, is time a phenomenon and does space have a materiality, or are they mathematical concepts ? A first response level is given by the impossibility to perform experiments about time and space.
\end{abstract}

Keywords: Definition; Terminology; Field Effect; Aging

\section{Introduction}

Some examples taken in several physics areas allow one to decide if it's possible to perform experiments about time and/or space. Given that time and space have no physical properties, are they observable, and can they be measured ? Can aging be observed ? Is relativistic covariance an experiment about time and space ? It must be emphasized that the basic rules of epistemology require the use of precise terminology.

\section{Two Basic Experiments}

Observation and measurement are no doubt the most basic experiments of all.

\section{What is Precisely observed, when one claims to see Time and Space?}

To observe a clock is not to observe time. Indeed, we see the clock hands move, but it's not time that we are seeing. When we listen to the ticking of a grandfather clock, we don't hear time. The flow of water should not be confused with the passage of time : to watch the water flow is not to watch time pass. It's confirmed by the definition of time : « Time is a concept corresponding to what separates two states of a system » [1]. To observe the objects in front of us or around us, is not to observe space ; we see these objects, but we don't see space as such. It's confirmed by the definition of space : «Space is a concept corresponding to what separates two systems » [1]. The immediate conclusion is that time as such and space as such can not be observed.

\section{What is Exactly Measured when one claims to Measure Time and Space?}

During an experiment, instead of time, we measure what separates two states of a system : we check what the clock does simultaneously; the result is called « duration of the experiment ». Changes, movements, evolutions, are not time. Instead of space, we measure what separates two systems. For example, the inspector does not measure the length of a footprint : instead, he measures what separates the two ends of the footprint; the result is called " length of the footprint ». The fatal mistakes are caused by the current confusions between changes and time, and between objects and space. We have introduced the expression « technical field effects ", to describe these wrong interpretations of reality [1]. These confusions are swept away by the indispensable use of definitions of time and space. Time as such and space as such are not measurable. Instead, measurements are about states and systems. We'll have an interesting confirmation with the relativistic covariance.

\section{The Relativitivistic Covariance}

We first need to make the différence between the chronological age and the biological age. The chronological age, commonly called " age », is how many years separates the birth from today. The biological age is a complex concept which take into account the physical and mental health status of the individual. Einstein [2] published his theory of Special Relativity in 1905 and his theory 
of General Relativity in 1916. They describe interactions between objects, of which speed is close to that of light. The parameters used to measure the states of these objects are no longer invariant, they are covariant. For example a traveller moving at $150,000 \mathrm{~km} / \mathrm{s}$

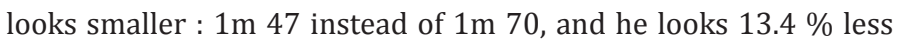
aged [1] ; « less aged » is related to his chronological age, not to his biological age. In fact, it's a kind of mathematical mirage. Indeed, the relativistic calculations show that it's just an appearance, and they allow the restoration of the exact data. Unfortunately, the stress caused by the relativistic travel has seriously aggravated the biological aging of the traveller ; therefore it led to an important increase of his biological age. To think that a relativistic travel make someone younger is a fantasy.

The mathematical invariance is restored by the introduction of the concept of space-time, which is an appropriate combination of space and time, and which can be defined : « The interval of spacetime is a concept corresponding to what separates two relativistic systems or two states of a relativistic system » [1]. Contrary to time and space, the interval of space-time is invariant. This definition confirms that the measurements are done on the states of the traveller. More broadly, the experiments are performed on the states of the relativistic systems, not on time or space. In General Relativity, which takes gravity into account, the interval of spacetime is represented by ten equations with ten unknowns. So far, there is no general solution; but the Universe is a special case which allow welcome simplifications [3].

\section{Action of Gravity}

Many experiments have been performed on clocks. During an experiment on an atomic clock in a Boing 747, the searchers were persuaded they were making experiments about time. In fact, gravity acts on clocks according to their technology : in microgravity a pendulum clock stops while an atomic clock becomes more precise [4]. It means clearly that experiments on clocks are not experiments about time as such. Ultimately, if the chronological age of the searchers changed at the same speed as their colleagues in the laboratory, their biological age was increased by the stress caused by the flight.

\section{Is Aging Observable and Measurable?}

Aging is not an increase in chronological age, but an increase in biological age ; it's not a phenomenon, but a concept. Here are two causes of aging, illustrated with the wrinkles and with the bacteria.

a) Wrinkles gradually appear when skin cells have exhausted their ability of scissiparity. This exhaustion results from the genetic heritage, the physical stresses and the epigenetic potentiation. Wrinkles are not caused by aging ; instead, they lead to aging. Therefore, wrinkles can be observed, not the aging of the skin. The wrinkles can be measured, not skin aging.

b) Bacteria Salmonella typhimurium is three times more virulent in microgravity [5] : it means that a population of bacteria age faster in microgavity. It's an experiment on bacteria, not an experiment on bacteria aging ; therefore it's not an experiment on time.

\section{Conclusion}

The definitions of time and space allow one to demonstrate that they can not be observed or measured. No experiments can be performed about time and space. It's not a surprise, because time and space are deprived of physical properties. Time is not a physical phenomenon and space has no materiality, they are mathematical concepts : they have no physical existence

\section{References}

1. Dassonville P (2012) The Invention of Time \& Space-Springer Verlag.

2. Einstein A (1916) Die Grundlage der allgemeinen Relativitätstheorie. Verlag von Johan Ambrosius Barth - Leipzig.

3. Mavridès S (1995) La Relativité-Presses Universitaires de France.

4. Hawking S (2005) A Briefer History of Time. New York : Bantam Dell, affiliate of Random House, Inc.

5. Sciences \& Avenir (2007).

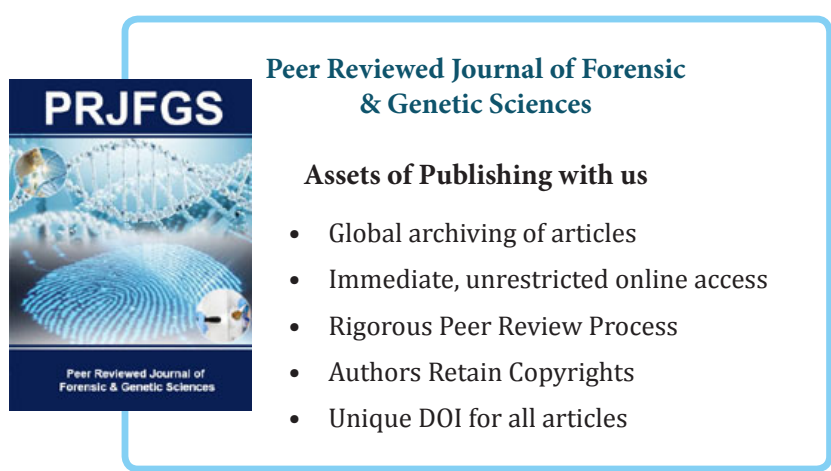

